\title{
Comparação entre GMFCS e CIF na avaliação da funcionalidade na paralisia cerebral
}

\author{
Comparison between GMFCS and ICF in the evaluation of \\ funcionality in cerebral palsy
}

\section{Comparación entre GMFCS y CIF en la evaluación de la funcionalidad en palsy cerebral}

\author{
Débora Taís Seifert Queiroz ${ }^{1}$, Arthur Cherem Netto Fernandes ${ }^{2}$, Murilo \\ Santos de Carvalho ${ }^{3}$, Guilherme Grivicich da Silva ${ }^{4}$, \\ Alessandra Bombarda Müller ${ }^{5}$
}

\begin{abstract}
1.Fisioterapeuta, Curso de Fisioterapia, Escola da Saúde, Unisinos, São Leopoldo-RS, Brasil. https://orcid.org/0000-0003-1499-4942

2.Fisioterapeuta, Curso de Fisioterapia, Escola da Saúde, Unisinos, São Leopoldo-RS, Brasil. https://orcid.org/0000-0002-2078-3802

3.Fisioterapeuta, Curso de Fisioterapia, Escola da Saúde, Unisinos, São Leopoldo-RS, Brasil. https://orcid.org/0000-0003-1862-4754

4.Fisioterapeuta, Mestre, docente do Curso de Fisioterapia, Escola da Saúde, Unisinos, São Leopoldo-RS, Brasil. https://orcid.org/0000-0002-8378-6349

5.Fisioterapeuta, Doutora, docente do Curso de Fisioterapia, Escola da Saúde, Unisinos, São Leopoldo-RS, Brasil. https://orcid.org/0000-0002-6256-9943
\end{abstract}

\begin{abstract}
Resumo
Introdução. A Paralisia Cerebral (PC) pode causar deformidades e movimentos atípicos que interferem na aquisição das funções motoras da criança. Objetivo. Foi comparar dois instrumentos mundialmente utilizados para avaliação da funcionalidade de crianças e adolescentes com PC. Método. Estudo realizado com 38 crianças com PC, de ambos os sexos, em fisioterapia semanalmente. Foram utilizados os instrumentos GMFCS e CIF-CJ para a avaliação da funcionalidade. Resultados. As crianças apresentaram: 68,4\% ( $n=26)$ comprometimento nos quatro membros (tetraparesia); $36,8 \%(n=14)$ comprometimento funcional grave com comorbidades associadas. Na comparação entre funcionalidade e funções do corpo, foram encontradas diferenças entre as cinco classificações funcionais e as funções intelectuais $(p=0,009)$, funções mentais da linguagem $(p=0,010)$, funções da mobilidade das articulações $(p<0,001)$, funções do tônus muscular $(p<0,001)$ e funções relacionadas ao controle dos movimentos voluntários $(p<0,001)$. Na comparação entre funcionalidade e atividades e participação, foram encontradas diferenças entre as cinco classificações funcionais e a manutenção da posição do corpo $(p<0,001)$, uso fino da mão $(p<0,001)$, andar $(p<0,001)$, deslocar-se por diferentes locais $(p<0,001)$, comer $(p=0,010)$, interações interpessoais básicas $(p=0,005)$ e relações familiares $(p=0,004)$. Quanto maior o comprometimento funcional, maior o grau de comprometimento da deficiência dessas crianças nas funções do corpo, atividades e participação e fatores ambientais. Conclusão. Os dois instrumentos são complementares na avaliação da criança com PC e podem respaldar o raciocínio clínico para tomada de decisões dos profissionais que os assistem, bem como nortear políticas públicas para a promoção da qualidade de vida dessa população.
\end{abstract}

Unitermos. Paralisia Cerebral; Classificação Internacional de Funcionalidade; Incapacidade e Saúde; Espasticidade Muscular

\footnotetext{
Abstract

Introduction. Cerebral Palsy (CP) can cause deformities and atypical movements that interfere with the acquisition of the child's motor functions. Objective. It was to compare two
} 
instruments used worldwide to assess the functionality of children and adolescents with CP. Method. Study carried out with 38 children with $\mathrm{CP}$, of both sexes, undergoing physical therapy weekly. The GMFCS and CIF-CJ instruments were used to assess functionality. Results. The children presented: $68.4 \%(n=26)$ impairment in the four limbs (tetraparesis); $36.8 \%(n=14)$ severe functional impairment with associated comorbidities. In the comparison between functionality and body functions, differences were found between the five functional classifications and intellectual functions $(p=0.009)$, mental functions of language $(p=0.010)$, functions of joint mobility $(p<0.001)$, functions of the muscle tone $(p<0.001)$ and functions related to the control of voluntary movements $(p<0.001)$. In the comparison between functionality and activities and participation, differences were found between the five functional classifications and the maintenance of the body position $(p<0.001)$, fine use of the hand $(p<0.001)$, walking $(p<0.001)$, moving by different locations $(p<0.001)$, eating $(p=0.010)$, basic interpersonal interactions $(p=0.005)$, and family relationships $(p=0.004)$. The greater the functional impairment, the greater the degree of impairment of these children's impairment in body functions, activities and participation and environmental factors. Conclusion. Both instruments are complementary in the assessment of children with CP and can support the clinical reasoning for decision-making by the professionals who assist them, as well as guide public policies to promote the quality of life of this population.

Keywords. Cerebral Palsy; International Classification of Functioning; Disability and Health; Muscle Spasticity

\section{Resumen}

Introducción. La Parálisis Cerebral (PC) puede causar deformidades y movimientos atípicos que interfieren con la adquisición de las funciones motoras del niño. Objetivo. Se trataba de comparar dos instrumentos utilizados a nivel mundial para evaluar la funcionalidad de niños y adolescentes con PC. Método. Estudio realizado con 38 niños con PC, de ambos sexos, sometidos a fisioterapia semanalmente. Se utilizaron los instrumentos GMFCS y CIF-CJ para evaluar la funcionalidad. Resultados. Los niños presentaron: 68,4\% $(n=26)$ deficiencia en los cuatro miembros (tetraparesia); $36,8 \%(n=14)$ deterioro funcional grave con comorbilidades asociadas. En la comparación entre funcionalidad y funciones corporales, se encontraron diferencias entre las cinco clasificaciones funcionales y funciones intelectuales $(p=0,009)$, funciones mentales del lenguaje $(p=0,010)$, funciones de movilidad articular $(p<0,001)$, funciones del tono muscular $(p<0,001)$ y funciones relacionadas con el control de los movimientos voluntarios $(p<0,001)$. En la comparación entre funcionalidad y actividades y participación, se encontraron diferencias entre las cinco clasificaciones funcionales y el mantenimiento de la posición corporal $(p<0,001)$, uso fino de la mano $(p<0,001)$, caminar $(p<0,001)$, moverse por diferentes localizaciones $(p<0,001)$, alimentación $(p=0,010)$, interacciones interpersonales básicas $(p=0,005)$ y relaciones familiares $(p=0,004)$. Cuanto mayor es el deterioro funcional, mayor es el grado de deterioro del deterioro de las funciones corporales, actividades y participación de estos niños y factores ambientales. Conclusión. Ambos instrumentos son complementarios en la evaluación de los niños con PC y pueden apoyar el razonamiento clínico para la toma de decisiones de los profesionales que los asisten, así como orientar políticas públicas para promover la calidad de vida de esta población.

Palabras clave. Parálisis Cerebral; Clasificación Internacional del Funcionamiento; de la Discapacidad y de la Salud; Espasticidad Muscular

Trabalho realizado na Escola da Saúde da Unisinos, São Leopoldo-RS, Brasil.

Conflito de interesse: não

Recebido em: $27 / 07 / 2020$

Aceito em: 05/10/2020

Endereço para correspondência: Alessandra B Müller. Av. Unisinos 950. Bairro Cristo Rei, São Leopoldo-RS, Brasil. Email: abombarda@unisinos.br 


\section{INTRODUÇÃO}

A Paralisia Cerebral (PC) é uma desordem do desenvolvimento que envolve distúrbios do movimento e da postura, podendo estar associada a déficits sensoriais e cognitivos, decorrente de uma lesão não progressiva causada no cérebro imaturo em desenvolvimento nos períodos pré-natal, perinatal e pós-natal ${ }^{1,2}$. É causa comum de deficiência física nos primeiros anos da infância, acompanhada de distúrbios sensoriais, percepção, cognição, comunicação, e aquisição de posturas inadequadas, ocasionando deformidades e movimentos atípicos que interferem na adequada aquisição das funções motoras durante o desenvolvimento ${ }^{3,4}$.

Uma consequência comum na PC é a diminuição das funções motoras grossas, identificada nas alterações dos padrões de movimento e na limitação da capacidade funcional. Se apresenta uni ou bilateralmente, nas formas espástica, discinética ou atáxica 5 .

Os recursos fisioterapêuticos utilizados em crianças com PC baseiam-se nos princípios da neuroplasticidade, da repetição de movimentos corretos, da correção postural, do fortalecimento e/ou alongamento muscular ${ }^{6}$. Assim, é necessário avaliar cuidadosamente a intensidade do tratamento para cada caso?.

A gravidade do comprometimento na criança com PC é extremamente variável, sendo categorizada por meio de classificações baseadas no movimento voluntário, de acordo com a faixa etária e o desempenho funcional da criança ${ }^{8}$. 0 
Sistema de Classificação da Função Motora Grossa (GMFCS) identifica cinco níveis de desempenho, desde a independência até graves limitações funcionais.

Ao preconizar a funcionalidade como uma condição de saúde, a Classificação Internacional de Funcionalidade, Incapacidade e Saúde (CIF) pode ser utilizada para descrever, organizar e compreender o impacto funcional da PC na vida da criança. A CIF promove uma linguagem internacional e comum entre profissionais de diferentes áreas do conhecimento e serve como marco conceitual para descrever os processos de funcionalidade e de incapacidade humana.

As alterações na função motora grossa acometem a maioria das crianças e adolescentes com PC, contribuindo para a diminuição da sua atividade e participação, assim como da sua capacidade funcional e do desempenho de suas atividades de vida diária, causando isolamento social, comorbidades e perda da qualidade de vida. Assim, a avaliação funcional da criança ou adolescente com PC tem como objetivo coletar o máximo de informações da atividade funcional, contemplando avaliação da estrutura e função do corpo, avaliação das atividades e participação, e assim, facilitar a determinação dos objetivos de tratamento.

No contexto de avaliação com foco na funcionalidade, identificar as condições de saúde de crianças com distúrbios motores é importante para o planejamento de ações que promovam sua autonomia e independência, favorecendo o entendimento das suas capacidades pelos familiares, 
cuidadores e equipe de saúde ${ }^{9}$. Assim, o objetivo deste trabalho foi comparar dois instrumentos mundialmente utilizados para avaliação da funcionalidade de crianças e adolescentes com PC que realizam fisioterapia em uma organização não governamental (ONG) da região metropolitana de Porto Alegre, RS.

\section{MÉTODO}

\section{Amostra}

Trata-se de um estudo transversal, de análise observacional, prospectivo, aprovado pelo Comitê de Ética em Pesquisa (CEP) da Universidade do Vale do Rio dos Sinos (Unisinos), sob parecer no 3.762.051. Todos os pais e/ou responsáveis das crianças e adolescentes que participaram assinaram o Termo de Consentimento Livre e Esclarecido (TCLE) e os dados foram coletados no mês de janeiro de 2020.

A população estudada foi composta por crianças e adolescentes com PC em acompanhamento fisioterapêutico na Associação Canoense de Deficientes Físicos (ACADEF), uma ONG sem fins lucrativos, de utilidade pública municipal, estadual e uma unidade de centro especializado em reabilitação (CER) nível III nas áreas física, auditiva e intelectual, situada na cidade de Canoas, Rio Grande do Sul (RS).

A amostra foi selecionada por conveniência, por meio de convite apresentado aos pais e/ou responsáveis. 
Participaram crianças e adolescentes de ambos os sexos, com diagnóstico de PC, com idade entre 0 e 18 anos, que realizavam fisioterapia semanalmente há, no mínimo, três semanas na ACADEF. Foram excluídos da pesquisa crianças e adolescentes cujos prontuários e/ou fichas de avaliação estavam incompletos, aqueles que estavam afastados dos atendimentos, em férias ou que não compareceram aos atendimentos.

\section{Procedimento}

Após identificação nos prontuários de quais crianças preenchiam os critérios de inclusão na pesquisa, foram coletados com os pais e/ou responsáveis dados de identificação, histórico prévio de saúde da criança e da gestação da mãe, questões relacionadas à funcionalidade, personalidade e atividades de participação.

Os atendimentos fisioterapêuticos realizados por profissional especialista foram observados. A funcionalidade foi avaliada utilizando o instrumento de classificação da função motora grossa Gross Motor Function Classification System (GMFCS) e a Classificação Internacional de Funcionalidade, Incapacidade e Saúde para Crianças e Jovens (CIF-CJ) resumida para crianças e jovens com PC.

O GMFCS permite avaliar crianças ou adolescentes até os 18 anos de idade, onde as descrições dos níveis são abrangentes e têm como função determinar qual nível melhor representa as habilidades e limitações na função motora grossa que a criança ou o adolescente apresentam. 
Os níveis são baseados nos movimentos iniciados voluntariamente, com ênfase no sentar-se, em transferências e em mobilidade ${ }^{10}$.

Esse sistema de classificação apresenta os seguintes níveis: crianças com pequena limitação da função e que deambulam sem restrições se enquadram no nível I (sem limitações) e nível II (com limitações); crianças que necessitam auxílio ou dispositivo manual de mobilidade (nível III); e os níveis que abrangem as crianças/adolescentes que utilizam tecnologia assistiva para locomoção: o nível IV caracterizado pela auto mobilidade com limitações e o nível $\mathrm{V}$, cujo indivíduo é transportado. ${ }^{5}$ Também é estruturado de acordo com as idades, sendo dividido em cinco fixas etárias: a primeira equivale ao período anterior ao aniversário de dois anos, a segunda entre o $2^{\circ}$ e 040 aniversário, a terceira entre $04^{\circ}$ e $06^{\circ}$ aniversário, a quarta entre o $6^{\circ}$ e $012^{\circ}$ aniversário e a quinta entre o $12^{\circ}$ e o $18^{\circ}$ aniversário ${ }^{10}$.

A CIF categoriza informações relacionadas aos processos de funcionalidade e de incapacidade, organizando e compreendendo o impacto positivo ou negativo da funcionalidade sob uma perspectiva biológica, individual e social. A CIF conta com códigos e qualificadores usados em combinações para classificar o problema que pode se referir a uma limitação, deficiência, restrição, barreira e facilitadores ${ }^{11}$.

No core set $\mathrm{CIF-C]}$ resumido para crianças e jovens com PC, os códigos são divididos em categorias: B corresponde à 
função do corpo; S, à estrutura do corpo; categoria D, à atividade e participação, e a categoria E corresponde a fatores ambientais. $O$ core set ainda apresenta $F P$, que corresponde aos fatores pessoais, item esse não classificado pela CIF $^{12,13}$. Já os qualificadores são responsáveis por informações especificas sobre funcionalidade, como sua localização, magnitude e natureza do problema. No primeiro momento se coloca o código da CIF e na sequência o qualificador separado por um sinal de + ou um ponto decimal, fechando assim a composição do código ${ }^{11}$ (Quadro 1).

Também foi utilizada a escala de Ashworth modificada para avaliação do tônus muscular, que apresenta seis graduações, onde 0 corresponde ao tônus normal e 4 corresponde às partes afetadas, rígidas à flexão ou extensão ${ }^{14}$.

\section{Análise Estatística}

Os dados foram agrupados, sintetizados e organizados de forma esquematizada no processador estatístico Statistical Package for the Social Science (SPSS), versão 21.0. A normalidade da amostra foi verificada via análise visual de histograma, sendo confirmada pelo teste ShapiroWilk. Na descrição da capacidade funcional, foi utilizada estatística descritiva com distribuição de frequência e porcentagem para as variáveis do tipo categóricas ordinais e medidas de tendência central (média/mediana) e de variabilidade (desvio padrão/amplitude interquartílica) para 
as variáveis do tipo quantitativas contínuas. Para a comparação entre as variáveis investigadas e o comprometimento funcional, foi utilizado o teste Quiquadrado. Foi adotado $p<0,05$.

Quadro 1. Códigos da CIF-CJ e seus descritores.

\begin{tabular}{|c|c|}
\hline Código da CIF-CJ & Descritor \\
\hline \multicolumn{2}{|c|}{ Influência das funções do corpo na funcionalidade } \\
\hline $\begin{array}{l}\text { B117 } \\
\text { B134 }\end{array}$ & $\begin{array}{l}\text { Funções intelectuais } \\
\text { Funções do sono }\end{array}$ \\
\hline B167 & Funções mentais da linguagem \\
\hline B210 & Funções da visão \\
\hline B280 & Sensação de dor \\
\hline B710 & Funções da mobilidade das articulações \\
\hline B735 & Funções do tônus muscular \\
\hline B760 & Funções relacionadas ao controle dos movimentos voluntários \\
\hline \multicolumn{2}{|c|}{ Influência das atividades e participação na funcionalidade } \\
\hline D415 & Manter a posição do corpo (sentada) \\
\hline D440 & Uso fino das mãos \\
\hline D450 & Andar \\
\hline D460 & Deslocar-se por diferentes locais \\
\hline $\begin{array}{l}\text { D530 } \\
\text { D550 } \\
\text { D710 }\end{array}$ & $\begin{array}{l}\text { Cuidados relacionados com o processo de excreção } \\
\text { Comer } \\
\text { Interações interpessoais básicas }\end{array}$ \\
\hline D760 & Relações familiares \\
\hline \multicolumn{2}{|c|}{ Influência da estrutura do corpo na funcionalidade } \\
\hline $\mathbf{S 1 1 0}$ & Estrutura do cérebro \\
\hline \multicolumn{2}{|c|}{ Influência de fatores ambientais na funcionalidade } \\
\hline E115 & Produtos e tecnologias para uso pessoal na vida diária \\
\hline E120 & $\begin{array}{l}\text { Produtos e tecnologias para mobilidade e o transporte pessoal em } \\
\text { ambientes internos e externos }\end{array}$ \\
\hline E125 & Produtos e tecnologias para comunicação \\
\hline E150 & $\begin{array}{l}\text { Produtos e tecnologias usados em projeto, arquitetura e construção de } \\
\text { edifícios para uso público }\end{array}$ \\
\hline E310 & Família imediata \\
\hline$E 320$ & Amigos \\
\hline E460 & Atividades sociais \\
\hline E580 & Serviços, sistemas e políticas de saúde \\
\hline \multicolumn{2}{|c|}{ Influência de fatores pessoais na funcionalidade } \\
\hline fp & Gosta de conversar e interagir \\
\hline
\end{tabular}




\section{RESULTADOS}

Foram avaliados 55 prontuários de crianças com diagnóstico de PC que estavam na agenda de atendimento fisioterapêutico da instituição. Um familiar não aceitou a participação do seu filho no estudo, três crianças foram excluídas da pesquisa por apresentarem ficha de avaliação com dados incompletos, outras três crianças foram afastadas por problemas de saúde, duas por estarem bloqueadas dos atendimentos e oito por falta justificada, resultando 38 pacientes incluídos na pesquisa.

A maioria da amostra $(n=22)$ era do sexo masculino (57,9\%), com média de idade de 4,8 2 2,6 anos, com idades que variaram entre 1 e 10 anos. A média da idade gestacional ao nascimento foi $33 \pm 5,3$ semanas. O tempo médio de internação na UTI foi $64,8 \pm 32,5$ dias, com variação entre 15 e 120 dias. A região topográfica mais comprometida foram os quatro membros (tetraparesia; $n=26 ; 68,4 \%$ ). Quando utilizado o GMFCS, identificou-se maior prevalência do comprometimento grau V $(n=14 ; 36,8 \%)$. As características da amostra estão descritas na Tabela 1.

A Tabela 2 apresenta as comparações entre os cinco grupos de classificação da funcionalidade associados à característica clínica dominante (tipo de PC), área topográfica de comprometimento, comorbidades e uso de medicamentos. Foi encontrada diferença na presença de hipotonia nos casos mais graves de comprometimento funcional $(p=0,001)$, a tetraparesia foi observada em todos os casos grau $\mathrm{V}(p<0,001)$, as comorbidades associadas 
foram mais prevalentes nos casos mais graves $(p=0,004)$ e as crianças menos comprometidas não faziam uso de medicações $(p=0,014)$.

Tabela 1. Caracterização geral da amostra.

\begin{tabular}{|c|c|c|}
\hline \multirow[t]{2}{*}{ Variáveis } & \multicolumn{2}{|c|}{ amostra $(n=38)$} \\
\hline & $\boldsymbol{n}$ & $\%$ \\
\hline \multicolumn{3}{|l|}{ Sexo } \\
\hline Masculino & 22 & 57,9 \\
\hline Feminino & 16 & 42,1 \\
\hline \multicolumn{3}{|l|}{ Raça } \\
\hline Branca & 27 & 71,1 \\
\hline Negra & 1 & 2,6 \\
\hline Parda & 10 & 26,3 \\
\hline \multicolumn{3}{|l|}{ Tipo de parto } \\
\hline Vaginal & 12 & 31,6 \\
\hline Cesariana & 24 & 63,1 \\
\hline Não respondeu & 2 & 5,3 \\
\hline \multicolumn{3}{|c|}{ Permaneceram na UTI } \\
\hline Não & 15 & 39,5 \\
\hline Sim & 23 & 60,5 \\
\hline \multicolumn{3}{|c|}{ Faz uso de medicação } \\
\hline Não & 19 & 50,0 \\
\hline Sim & 19 & 50,0 \\
\hline \multicolumn{3}{|c|}{ Característica clínica dominante } \\
\hline \multicolumn{3}{|c|}{ Hipotônica } \\
\hline Não & 20 & 25,6 \\
\hline Sim & 18 & 47,4 \\
\hline \multicolumn{3}{|l|}{ Discinética } \\
\hline Não & 31 & 81,6 \\
\hline Sim & 7 & 18,4 \\
\hline \multicolumn{3}{|l|}{ Espástica } \\
\hline Não & 1 & 2,6 \\
\hline Sim & 37 & 97,4 \\
\hline \multicolumn{3}{|l|}{ Área topográfica } \\
\hline Diparesia & 8 & 21,1 \\
\hline Hemiparesia & 4 & 10,5 \\
\hline Tetraparesia & 26 & 68,4 \\
\hline \multicolumn{3}{|c|}{ Comorbidades associadas } \\
\hline Não & 14 & 36,8 \\
\hline Sim & 24 & 63,2 \\
\hline \multicolumn{3}{|l|}{ GMFCS } \\
\hline Grau I & 7 & 18,4 \\
\hline Grau II & 3 & 7,9 \\
\hline Grau III & 5 & 13,2 \\
\hline Grau IV & 9 & 23,7 \\
\hline Grau V & 14 & 36,8 \\
\hline
\end{tabular}


Tabela 2. Comparações entre a classificação da funcionalidade (GMFCS) e tipo de PC, área topográfica, comorbidades associadas e uso de medicamentos.

\begin{tabular}{|c|c|c|c|c|c|c|c|c|c|c|c|}
\hline \multirow{3}{*}{ Variáreis } & \multicolumn{11}{|c|}{ GMFCS } \\
\hline & \multicolumn{2}{|c|}{ I $(n=7)$} & \multicolumn{2}{|c|}{ II $(n=3)$} & \multicolumn{2}{|c|}{$\begin{array}{c}\text { III } \\
(n=5)\end{array}$} & \multicolumn{2}{|c|}{$\begin{array}{c}\text { IV } \\
(n=9)\end{array}$} & \multicolumn{2}{|c|}{ V $(n=14)$} & \multirow[t]{2}{*}{$\boldsymbol{p}$} \\
\hline & $n$ & $\%$ & $n$ & $\%$ & $n$ & $\%$ & $n$ & $\%$ & $n$ & $\%$ & \\
\hline Sexo & & & & & & & & & & & 0,534 \\
\hline Masculino & 3 & 42,9 & 1 & 33,3 & 2 & 40,0 & 7 & 77,8 & 9 & 64,3 & \\
\hline Feminino & 4 & 57,1 & 2 & 66,7 & 3 & 60,0 & 2 & 22,2 & 5 & 35,7 & \\
\hline Hipotonia & & & & & & & & & & & 0,001 \\
\hline Não & 7 & 100,0 & 3 & 100,0 & 3 & 60,0 & 4 & 44,4 & 3 & 21,4 & \\
\hline Sim & & & & & 2 & 40,0 & 5 & 55,6 & 11 & 78,6 & \\
\hline Discinesia & & & & & & & & & & & 0,726 \\
\hline Não & 6 & 85,7 & 3 & 100,0 & 3 & 60,0 & 8 & 88,9 & 11 & 78,6 & \\
\hline Sim & 1 & 14,3 & & & 2 & 40,0 & 1 & 11,1 & 3 & 21,4 & \\
\hline Espasticidade & & & & & & & & & & & 0,999 \\
\hline Não & & & & & & & & & 1 & 7,1 & \\
\hline Sim & 7 & 100,0 & 3 & 100,0 & 5 & 100,0 & 9 & 100,0 & 13 & 92,9 & \\
\hline $\begin{array}{l}\text { Área } \\
\text { topográfica }\end{array}$ & & & & & & & & & & & $<0,001$ \\
\hline Diparesia & 4 & 57,1 & 1 & 33,3 & 2 & 40,0 & 1 & 11,1 & & & \\
\hline Hemiparesia & 3 & 42,9 & 2 & 66,7 & 1 & 20,0 & 8 & 88,9 & & & \\
\hline Tetraparesia & & & & & 2 & 40,0 & & & 14 & 100,0 & \\
\hline $\begin{array}{l}\text { Comorbidades } \\
\text { associadas }\end{array}$ & & & & & & & & & & & 0,004 \\
\hline Não & 5 & 71,4 & 3 & 100,0 & 1 & 20,0 & 4 & 44,4 & 1 & 7,1 & \\
\hline Sim & 2 & 28,6 & & & 4 & 80,0 & 5 & 55,6 & 13 & 92,9 & \\
\hline \multicolumn{12}{|l|}{$\begin{array}{l}\text { Tipo de } \\
\text { comorbidade }\end{array}$} \\
\hline Convulsão & & & & & & & 2 & 22,2 & 6 & 42,9 & 0,637 \\
\hline $\begin{array}{l}\text { Déficit } \\
\text { cognitivo }\end{array}$ & 2 & 28,6 & & & 2 & 40,0 & & - & 6 & 42,9 & 0,055 \\
\hline Epilepsia & 1 & 14,3 & & & & & 2 & 22,2 & 2 & 14,3 & 0,367 \\
\hline Outras & 1 & 14,3 & & & 2 & 40,0 & 1 & 11,1 & 2 & 14,3 & 0,615 \\
\hline Medicações & & & & & & & & & & & 0,014 \\
\hline Não & 7 & 100,0 & 2 & 66,7 & 3 & 60,0 & 3 & 33,3 & 4 & 28,6 & \\
\hline Sim & & & 1 & 33,3 & 2 & 40,0 & 6 & 66,7 & 10 & 71,4 & \\
\hline
\end{tabular}

Também foram comparadas as distribuições da amostra entre os cinco grupos de classificação da funcionalidade (GMFCS) e o core set CIF-CJ resumido para crianças e jovens com PC, que engloba as categorias da CIF funções do corpo (B), estruturas do corpo (S), atividades e participação (D) e fatores ambientais e pessoais $(E)$. 
$\mathrm{Na}$ comparação entre funcionalidade (GMFCS) e funções do corpo (B), foram encontradas diferenças entre as cinco classificações funcionais e as funções intelectuais (B117; $p=0,009)$, funções mentais da linguagem (B167; $p=0,010$ ), funções da mobilidade das articulações (B710; $p<0,001)$, funções do tônus muscular (B735; $p<0,001)$ e funções relacionadas ao controle dos movimentos voluntários (B760; $p<0,001)$, onde quanto maior $\mathrm{o}$ comprometimento funcional identificado pela classificação GMFCS, maior o grau de comprometimento da deficiência dessas crianças, como visualizado na Tabela 3.

Na comparação entre a categoria da CIF estruturas do corpo (S) e a classificação da funcionalidade (GMFCS), não foram encontradas diferenças entre os cinco grupos de classificação da funcionalidade nesta amostra.

$\mathrm{Na}$ comparação entre funcionalidade (GMFCS) e atividades e participação (D), foram encontradas diferenças entre as cinco classificações funcionais e a manutenção da posição do corpo (D415; $p<0,001$ ), uso fino da mão (D440; $p<0,001)$, andar (D450; $p<0,001)$, deslocar-se por diferentes locais (D460; $p<0,001)$, comer (D550; $p=0,010)$, interações interpessoais básicas ( $710 ; p=0,005)$ e relações familiares (D760; $p=0,004)$, onde quanto maior 0 comprometimento funcional (maior grau na GMFCS), maiores eram as dificuldades apresentadas (ou relatadas) pelas crianças desta amostra nessas atividades e participações (Tabela 4). 
Tabela 3. Comparações entre função do corpo (CIF) e classificação da funcionalidade (GMFCS).

\begin{tabular}{|c|c|c|c|c|c|c|c|c|c|c|c|}
\hline \multirow{3}{*}{ CIF } & \multicolumn{11}{|c|}{ GMFCS } \\
\hline & \multicolumn{2}{|c|}{ I $(n=7)$} & \multicolumn{2}{|c|}{ II $(n=3)$} & \multicolumn{2}{|c|}{$\begin{array}{c}\text { III } \\
(n=5)\end{array}$} & \multicolumn{2}{|c|}{$\begin{array}{c}\text { IV } \\
(n=9)\end{array}$} & \multicolumn{2}{|c|}{$\begin{array}{c}V \\
(n=14)\end{array}$} & \multirow{2}{*}{$\boldsymbol{p}$} \\
\hline & $n$ & $\%$ & $n$ & $\%$ & $n$ & $\%$ & $n$ & $\%$ & $n$ & $\%$ & \\
\hline B 117 & & & & & & & & & & & 0,009 \\
\hline Nenhuma & 3 & 42,9 & 3 & 100,0 & & & 1 & 11,1 & & & \\
\hline Ligeira & 2 & 28,6 & & & 2 & 40,0 & 2 & 22,2 & 1 & 7,1 & \\
\hline Moderada & 2 & 28,6 & & & 2 & 40,0 & 3 & 33,3 & 4 & 28,6 & \\
\hline Grave & & & & & 1 & 20,0 & 3 & 33,3 & 4 & 28,6 & \\
\hline Completa & & & & & & & & & 5 & 35,7 & \\
\hline B 134 & & & & & & & & & & & 0,388 \\
\hline Nenhuma & 7 & 100,0 & 2 & 66,7 & 2 & 40,0 & 6 & 66,7 & 10 & 71,4 & \\
\hline Moderada & & & 1 & 33,3 & 1 & 20,0 & 2 & 22,2 & 1 & 7,1 & \\
\hline Grave & & & & & 2 & 40,0 & 1 & 11,1 & 1 & 7,1 & \\
\hline Completa & & & & & & & & & 2 & 14,3 & \\
\hline B 167 & & & & & & & & & & & 0,010 \\
\hline Nenhuma & 3 & 42,9 & 1 & 33,3 & & & 1 & 11,1 & & & \\
\hline Ligeira & 1 & 14,3 & 2 & 66,7 & & & 1 & 11,1 & 1 & 7,1 & \\
\hline Moderada & 2 & 28,6 & & & 3 & 60,0 & 3 & 33,3 & 1 & 7,1 & \\
\hline Grave & 1 & 14,3 & & & 2 & 40,0 & 4 & 44,4 & 7 & 50,0 & \\
\hline Completa & & & & & & & & & 5 & 35,7 & \\
\hline B 210 & & & & & & & & & & & 0,112 \\
\hline Nenhuma & 4 & 57,1 & 1 & 33,3 & 4 & 80,0 & 3 & 33,3 & 9 & 64,3 & \\
\hline Ligeira & 2 & 28,6 & 1 & 33,3 & & & & & & & \\
\hline Moderada & & & 1 & 33,3 & & & 4 & 44,4 & 1 & 7,1 & \\
\hline Grave & 1 & 14,3 & & & 1 & 20,0 & 2 & 22,2 & 4 & 28,6 & \\
\hline B 280 & & & & & & & & & & & 0,476 \\
\hline Nenhuma & 6 & 85,7 & 3 & 100,0 & 4 & 80,0 & 5 & 55,6 & 7 & 50,0 & \\
\hline Moderada & 1 & 14,3 & & & & & 3 & 33,3 & 6 & 42,9 & \\
\hline Grave & & & & & 1 & 20,0 & 1 & 11,1 & 1 & 7,1 & \\
\hline B 710 & & & & & & & & & & & $<0,001$ \\
\hline Ligeira & 5 & 71,4 & & & & & & & & & \\
\hline Moderada & 2 & 28,6 & 3 & 100,0 & 3 & 60,0 & 1 & 11,1 & 1 & 7,1 & \\
\hline Grave & & & & & 2 & 40,0 & 7 & 77,8 & 9 & 64,3 & \\
\hline Completa & & & & & & & 1 & 11,1 & 4 & 28,6 & \\
\hline B 735 & & & & & & & & & & & $<0,001$ \\
\hline Ligeira & 5 & 71,4 & & & & & & & & & \\
\hline Moderada & 2 & 28,6 & 3 & 100,0 & 3 & 60,0 & 1 & 11,1 & 1 & 7,1 & \\
\hline Grave & & & & & 2 & 40,0 & 7 & 77,8 & 10 & 71,4 & \\
\hline Completa & & & & & & & 1 & 11,1 & 3 & 21,4 & \\
\hline B 760 & & & & & & & & & & & $<0,001$ \\
\hline Nenhuma & 4 & 57,1 & & & & & & & & & \\
\hline Ligeira & 2 & 28,6 & 3 & 100,0 & & & & & & & \\
\hline Moderada & 1 & 14,3 & & & 3 & 60,0 & 3 & 33,3 & 2 & 14,3 & \\
\hline Grave & & & & & 2 & 40,0 & 6 & 66,7 & 5 & 35,7 & \\
\hline Completa & & & & & & & & & 7 & 50,0 & \\
\hline
\end{tabular}

B: funções do corpo. 
Tabela 4. Comparações entre atividades e participação (CIF) e classificação da funcionalidade (GMFCS).

\begin{tabular}{|c|c|c|c|c|c|c|c|c|c|c|c|}
\hline \multirow{3}{*}{ CIF } & \multicolumn{11}{|c|}{ GMFCS } \\
\hline & \multicolumn{2}{|c|}{$\begin{array}{c}\mathbf{I} \\
(n=7)\end{array}$} & \multicolumn{2}{|c|}{ II $(n=3)$} & \multicolumn{2}{|c|}{$\begin{array}{c}\text { III } \\
(n=5)\end{array}$} & \multicolumn{2}{|c|}{$\begin{array}{c}\text { IV } \\
(n=9)\end{array}$} & \multicolumn{2}{|c|}{$\begin{array}{c}V \\
(n=14)\end{array}$} & \multirow{2}{*}{$\boldsymbol{p}$} \\
\hline & $n$ & $\%$ & $n$ & $\%$ & $n$ & $\%$ & $n$ & $\%$ & $n$ & $\%$ & \\
\hline D 415 & & & & & & & & & & & $<0,001$ \\
\hline Nenhuma & 6 & 85,7 & 3 & 100,0 & & & & & & & \\
\hline Ligeira & 1 & 14,3 & & & 4 & 80,0 & & & & & \\
\hline Moderada & & & & & 1 & 20,0 & 6 & 66,7 & 1 & 7,1 & \\
\hline Grave & & & & & & & 3 & 33,3 & 5 & 35,7 & \\
\hline Completa & & & & & & & & & 8 & 57,1 & \\
\hline D 440 & & & & & & & & & & & $<0,001$ \\
\hline Nenhuma & 4 & 57,1 & 3 & 100,0 & 1 & 20,0 & 1 & 11,1 & & & \\
\hline Ligeira & 1 & 14,3 & & & 2 & 40,0 & 3 & 33,3 & & & \\
\hline Moderada & 2 & 28,6 & & & 1 & 20,0 & 4 & 44,4 & 1 & 7,1 & \\
\hline Grave & & & & & 1 & 20,0 & 1 & 11,1 & 9 & 64,3 & \\
\hline Completa & & & & & & & & & 4 & 28,6 & \\
\hline D 450 & & & & & & & & & & & $<0,001$ \\
\hline Ligeira & 5 & 71,4 & & & & & 1 & 11,1 & & & \\
\hline Moderada & 2 & 28,6 & 3 & 100,0 & 3 & 60,0 & 1 & 11,1 & & & \\
\hline Grave & & & & & 2 & 40,0 & 7 & 77,8 & 2 & 14,3 & \\
\hline Completa & & & & & & & & & 12 & 85,7 & \\
\hline D 460 & & & & & & & & & & & $<0,001$ \\
\hline Ligeira & 3 & 42,9 & & & & & & & & & \\
\hline Moderada & 3 & 42,9 & 2 & 66,7 & 2 & 40,0 & 2 & 22,2 & & & \\
\hline Grave & 1 & 14,3 & 1 & 33,3 & 3 & 60,0 & 7 & 77,8 & 1 & 7,1 & \\
\hline Completa & & & & & & & & & 13 & 92,9 & \\
\hline D 530 & & & & & & & & & & & 0,076 \\
\hline Nenhuma & 4 & 57,1 & 1 & 33,3 & 2 & 40,0 & 1 & 11,1 & & & \\
\hline Ligeira & & & & & & & & & 1 & 7,1 & \\
\hline Grave & 3 & 42,9 & 2 & 66,7 & 3 & 60,0 & 8 & 88,9 & 10 & 71,4 & \\
\hline Completa & & & & & & & & & 3 & 21,4 & \\
\hline D 550 & & & & & & & & & & & 0,001 \\
\hline Nenhuma & 3 & 42,9 & 3 & 100,0 & 1 & 20,0 & 2 & 22,2 & 1 & 7,1 & \\
\hline Ligeira & 2 & 28,6 & & & & & & & & & \\
\hline Moderada & 1 & 14,3 & & & 2 & 40,0 & 4 & 44,4 & & & \\
\hline Grave & 1 & 14,3 & & & 2 & 40,0 & 3 & 33,3 & 7 & 50,0 & \\
\hline Completa & & & & & & & & & 6 & 42,9 & \\
\hline D 710 & & & & & & & & & & & 0,005 \\
\hline Nenhuma & 3 & 42,9 & 1 & 33,3 & & & 1 & 11,1 & & & \\
\hline Ligeira & 1 & 14,3 & 2 & 66,7 & 2 & 40,0 & 2 & 22,2 & 1 & 7,1 & \\
\hline Moderada & 3 & 42,9 & & & 2 & 40,0 & 3 & 33,3 & 1 & 7,1 & \\
\hline Grave & & & & & 1 & 20,0 & 3 & 33,3 & 9 & 64,3 & \\
\hline Completa & & & & & & & & & 3 & 21,4 & \\
\hline D 760 & & & & & & & & & & & 0,004 \\
\hline Nenhuma & 3 & 42,9 & 2 & 66,7 & 3 & 60,0 & 1 & 11,1 & 1 & 7,1 & \\
\hline Ligeira & 3 & 42,9 & 1 & 33,3 & 1 & 20,0 & 1 & 11,1 & & & \\
\hline Moderada & 1 & 14,3 & & & & & 7 & 77,8 & 7 & 50,0 & \\
\hline Grave & & & & & & & & & 5 & 35,7 & \\
\hline Completa & & & & & 1 & 20,0 & & & 1 & 7,1 & \\
\hline
\end{tabular}

D: atividades e participação 
Na comparação entre funcionalidade (GMFCS) e fatores ambientais (E), foram encontradas diferenças entre as cinco classificações funcionais e produtos e tecnologias para uso pessoal na vida diária (E115; $p=0,006)$, produtos e tecnologias para mobilidade e transporte pessoal em ambientes internos e externos (E120; $p<0,001)$, e produtos e tecnologias usados em projetos, arquitetura e construção de edifícios para uso público ( $\mathrm{E} 150 ; p=0,010)$, onde quanto maior o comprometimento funcional, mais completos e graves eram os facilitadores (Tabela 5).

Na comparação entre funcionalidade (GMFCS) e fatores pessoais (FP*), associados à curiosidade da criança, pode ser observado que das 14 crianças classificadas como GMFCS V (maior grau de comprometimento funcional), 7 (50\%) pontuaram negativo, o que pode estar associado com 0 comprometimento das funções intelectuais (B117) e funções mentais da linguagem (B167) nestas crianças.

\section{DISCUSSÃO}

O objetivo deste trabalho foi comparar a classificação funcional de crianças e adolescentes com PC utilizando os instrumentos GMFCS e CIF-CJ.

O sexo masculino foi o mais prevalente nesta amostra $(57,9 \%)$, assim como em estudos prévios que apontaram maior prevalência de participantes do sexo masculino (mais de $70 \%$ ) dentre todas as idades avaliadas ${ }^{15,16}$. 
Tabela 5. Comparações entre fatores ambientais (CIF) e classificação da funcionalidade (GMFCS).

\begin{tabular}{|c|c|c|c|c|c|c|c|c|c|c|c|}
\hline \multirow{3}{*}{ CIF } & \multicolumn{11}{|c|}{ GMFCS } \\
\hline & \multicolumn{2}{|c|}{ I $(n=7)$} & \multicolumn{2}{|c|}{ II $(n=3)$} & \multicolumn{2}{|c|}{$\begin{array}{c}\text { III } \\
(n=5)\end{array}$} & \multicolumn{2}{|c|}{ IV $(n=9)$} & \multicolumn{2}{|c|}{$\begin{array}{c}V \\
(n=14)\end{array}$} & \multirow{2}{*}{$p$} \\
\hline & $n$ & $\%$ & $n$ & $\%$ & $n$ & $\%$ & $n$ & $\%$ & $n$ & $\%$ & \\
\hline E 115 & & & & & & & & & & & 0,006 \\
\hline $1+$ & 1 & 14,3 & & & & & & & & & \\
\hline $2+$ & 3 & 42,9 & 2 & 66,7 & 1 & 20,0 & & & & & \\
\hline $3+$ & 2 & 28,6 & 1 & 33,3 & 3 & 60,0 & 3 & 33,3 & 3 & 21,4 & \\
\hline $4+$ & 1 & 14,3 & & & 1 & 20,0 & 6 & 66,7 & $\begin{array}{l}1 \\
1\end{array}$ & 78,6 & \\
\hline E 120 & & & & & & & & & & & $<0,001$ \\
\hline 0 & 5 & 71,4 & 1 & 33,3 & & & & & & & \\
\hline $1+$ & 1 & 14,3 & & & & & & & & & \\
\hline 2 & 1 & 14,3 & & & 1 & 20,0 & 1 & 11,1 & 2 & 14,3 & \\
\hline $2+$ & & & 1 & 33,3 & 3 & 60,0 & & & & & \\
\hline 3 & & & & & & & 1 & 11,1 & 1 & 7,1 & \\
\hline $3+$ & & & 1 & 33,3 & 1 & 20,0 & 7 & 77,8 & 5 & 35,7 & \\
\hline $4+$ & & & & & & & & & 6 & 42,9 & \\
\hline E 125 & & & & & & & & & & & 0,104 \\
\hline 0 & 3 & 42,9 & 2 & 66,7 & & & & & 1 & 7,1 & \\
\hline $1+$ & & & & & 2 & 40,0 & 1 & 11,1 & 5 & 35,7 & \\
\hline 2 & & & 1 & 33,3 & 2 & 40,0 & & & 1 & 7,1 & \\
\hline $2+$ & 3 & 42,9 & & & & 7 & 77,8 & & 3 & 21,4 & \\
\hline 3 & & & & & 1 & 20,0 & & & 3 & 21,4 & \\
\hline $3+$ & 1 & 14,3 & & & & & 1 & 11,1 & 1 & 7,1 & \\
\hline E 150 & & & & & & & & & & & 0,019 \\
\hline 0 & 1 & 14,3 & & & & & & & & & \\
\hline 1 & 1 & 14,3 & & & & & & & & & \\
\hline $1+$ & 3 & 42,9 & 1 & 33,3 & 1 & 20,0 & & & 3 & 21,4 & \\
\hline 2 & & & 1 & 33,3 & & & & & & & \\
\hline $2+$ & 2 & 28,6 & & & 4 & 80,0 & 8 & 88,9 & 9 & 64,3 & \\
\hline 3 & & & & & & & 1 & 11,1 & & & \\
\hline $3+$ & & & 1 & 33,3 & & & & & 2 & 14,3 & \\
\hline E 310 & & & & & & & & & & & 0,745 \\
\hline $1+$ & & & 1 & 33,3 & & & & & & & \\
\hline 2 & 1 & 14,3 & & & & & 1 & 11,1 & 2 & 14,3 & \\
\hline $2+$ & & & & & & & & & 1 & 7,1 & \\
\hline 3 & 1 & 14,3 & & & & & 1 & 11,1 & & & \\
\hline $3+$ & 4 & 57,1 & 2 & 66,7 & 4 & 80,0 & 5 & 55,6 & 8 & 57,1 & \\
\hline 4 & & & & & 1 & 20,0 & & & & & \\
\hline $4+$ & 1 & 14,3 & & & & & 2 & 22,2 & 3 & 21,4 & \\
\hline E 320 & & & & & & & & & & & 0,416 \\
\hline 1 & & & & & & & 1 & 11,1 & & & \\
\hline $1+$ & & & & & & & 1 & 11,1 & & & \\
\hline 2 & 3 & 42,9 & & & 2 & 40,0 & 3 & 33,3 & 8 & 57,1 & \\
\hline $2+$ & 3 & 42,9 & 3 & 100,0 & 2 & 40,0 & 4 & 44,4 & 3 & 21,4 & \\
\hline 3 & & & & & 1 & 20,0 & & & 2 & 14,3 & \\
\hline $3+$ & 1 & 14,3 & & & & & & & & & \\
\hline 4 & & & & & & & & & 1 & 7,1 & \\
\hline
\end{tabular}

E: fatores ambientais. 
Tabela 5 (cont.). Comparações entre fatores ambientais (CIF) e classificação da funcionalidade (GMFCS).

\begin{tabular}{|c|c|c|c|c|c|c|c|c|c|c|c|}
\hline \multirow{3}{*}{ CIF } & \multicolumn{11}{|c|}{ GMFCS } \\
\hline & \multicolumn{2}{|c|}{ I $(n=7)$} & \multicolumn{2}{|c|}{ II $(n=3)$} & \multicolumn{2}{|c|}{$\underset{(n=5)}{\text { III }}$} & \multicolumn{2}{|c|}{ IV $(n=9)$} & \multicolumn{2}{|c|}{$\begin{array}{c}V \\
(n=14)\end{array}$} & \multirow{2}{*}{$\boldsymbol{p}$} \\
\hline & $n$ & $\%$ & $n$ & $\%$ & $n$ & $\%$ & $n$ & $\%$ & $n$ & $\%$ & \\
\hline E 460 & & & & & & & & & & & 0,066 \\
\hline 1 & 1 & 14,3 & & & & & & & & & \\
\hline $1+$ & 1 & 14,3 & 1 & 33,3 & 1 & 20,0 & & & 2 & 14,3 & \\
\hline 2 & & & & & & & 1 & 11,1 & & & \\
\hline $2+$ & 2 & 28,6 & 1 & 33,3 & 1 & 20,0 & 3 & 33,3 & & & \\
\hline 3 & 1 & 14,3 & & & 2 & 40,0 & & 11,1 & 3 & 21,4 & \\
\hline $3+$ & 2 & 28,6 & 1 & 33,3 & & & & & & & \\
\hline 4 & & & & & 1 & 20,0 & 4 & 44,4 & 9 & 64,3 & \\
\hline E 580 & & & & & & & & & & & 0,221 \\
\hline $2+$ & 1 & 14,3 & 2 & 66,7 & 1 & 20,0 & & & 1 & 7,1 & \\
\hline $3+$ & 6 & 85,7 & 1 & 33,3 & 4 & 80,0 & 9 & 100,0 & $\begin{array}{l}1 \\
2\end{array}$ & 85,7 & \\
\hline $4+$ & & & & & & & & & 1 & 7,1 & \\
\hline
\end{tabular}

E: fatores ambientais.

Estudos de incidência e prevalência da população com PC no cenário nacional são inconsistentes, entretanto, 0 censo demográfico de 2010 indicava 45.606 .048 pessoas com algum tipo de deficiência relatada, como a visual, a auditiva, a motora e a intelectual, constituindo $23,9 \%$ da população ${ }^{17}$.

A presença de hipotonia também esteve associada ao pior comprometimento funcional das crianças desta amostra, cuja média de idade gestacional ao nascimento foi 33,5 semanas, o que caracteriza recém-nascido prematuro (RNPT). Em comparação com os recém-nascidos a termo, os RNPT demonstraram ter um repertório motor particular, caracterizado principalmente por déficits no tônus muscular, no controle postural e no equilíbrio e ativação muscular 
contra a gravidade, o que pode explicar as diferenças encontradas com relação ao desempenho motor $^{18}$.

Quanto à área topográfica da PC, pode-se identificar que todas aquelas crianças que apresentavam algum comprometimento nos quatro membros do corpo (tetraparesia) foram classificadas como grau $V$ (pior comprometimento) na GMFCS. Pesquisas prévias que avaliaram a viabilidade de aplicar um programa de intervenção diária de fisioterapia em crianças com PC do tipo tetraparéticas nível V na GMFCS, e que avaliaram 147 crianças com suspeita de asfixia ao nascimento, onde $41 \%$ apresentaram encefalopatia neonatal moderada ou grave, associam os maiores níveis de GMFCS (IV e V) à condição de tetraparesia espástica. Essas pesquisas relataram que a reduzida experiência sensório-motora e o precário controle postural dessas crianças mais comprometidas limitam sua capacidade de adaptação e variabilidade de movimento ${ }^{19,20}$.

As crianças que apresentaram algum tipo de comorbidade associada foram classificadas com maior grau de comprometimento funcional (das 14 crianças com GMFCS V, 13 (92,9\%) apresentaram algum tipo de comorbidade) e maior necessidade de uso de medicações, bem como história de maior tempo de internação hospitalar após o nascimento. Este achado se assemelha ao encontrado no estudo que, investigando as condições socioeconômicas e de saúde, exame dos sinais vitais, avaliação pneumofuncional e classificação da função motora grossa de crianças com PC, indica que as crianças que apresentavam maior nível de 
comprometimento na GMFCS (nível V), também apresentavam mais comorbidades associadas ${ }^{21}$.

O maior comprometimento funcional, relacionado à alteração de tônus, encurtamentos musculares e deformidades ósseas em membros superiores e membros inferiores, pode ser decorrente da maior imobilidade no leito ou na cadeira de rodas e a inviabilidade do ortostatismo, condições que, com o passar do tempo, ocasionam alterações na mecânica respiratória, disfunções no trato urinário e na condição óssea, por exemplo ${ }^{18}$.

Neste estudo, na comparação entre a classificação da funcionalidade pelo instrumento GMFCS e as categorias descritas pelo modelo da CIF, pode-se observar que os códigos do core set da CIF-CJ resumido para crianças e jovens com PC estão associados aos níveis de funcionalidade apresentados pelo GMFCS.

Pesquisadores analisaram 140 prontuários de crianças com PC, e utilizaram códigos da CIF relacionados a funções do corpo e atividades e participação. Como no presente estudo, os autores observaram diferenças estatisticamente significativas na comparação entre os diferentes níveis funcionais, apontando que, quanto maior o nível GMFCS (pior classificação funcional), menor o desempenho da função motora grossa e pior funcionalidade demonstrada no core set da CIF-CJ ${ }^{22}$. Estes achados também foram relatados no estudo que verificou a qualidade motora e a capacidade funcional de 17 crianças com PC, onde a maioria dos 
participantes $(70,6 \%)$ apresentou níveis mais graves de comprometimento na GMFCS ${ }^{23}$.

Ao comparar funções do corpo e GMFCS, observou-se maior comprometimento nas funções intelectuais, funções mentais de linguagem, mobilidade das articulações, tônus muscular e controle dos movimentos voluntários nas crianças com maior escore na GMFCS, possivelmente devido ao comprometimento sensorial e de comunicação, assim como as disfunções funcionais do movimento, tanto em membros superiores quanto inferiores encontrados na amostra deste estudo.

Na comparação entre a categoria da CIF estruturas do corpo (S) e a classificação da funcionalidade (GMFCS), não foram encontradas diferenças significantes entre os cinco grupos de classificação da funcionalidade nesta amostra, porque quase todas as crianças apresentaram qualificador 8 (não especificada). Reporta-se a falta de informação e conhecimento dos pais e/ou responsáveis, pois a grande maioria não sabia descrever a estrutura comprometida.

Neste estudo, as crianças com maior escore no GMFCS (pior funcionalidade) apresentaram mais dificuldades associadas a atividades e participação. Pesquisa prévia que avaliou crianças com PC também aponta que aquelas que apresentaram maior escore no GMFCS, apresentaram importantes limitações relacionadas principalmente ao desempenho de suas atividades e restrições na sua participação, além dos aspectos de mobilidade e relações 
sociais no dia-a-dia, impactando também na comunicação e aquisições sensório-motoras ${ }^{24}$.

Foi encontrada maior necessidade de produtos e tecnologias para uso pessoal na vida diária das crianças desta pesquisa, assim como a mobilidade e o transporte pessoal em ambientes internos e externos, tal como a acessibilidade a edifícios de uso público. Quanto maior o escore no GMFCS, mais completo e grave foram os facilitadores e barreiras. Muitas dessas crianças possuem algum tipo de auxílio para locomoção, utilizam algum meio de transporte para se locomover, assim como têm acesso a locais públicos, considerando que são assistidas por uma ONG que atua na habilitação e reabilitação da pessoa com deficiência $(P C D)$ e na promoção de sua integração à vida comunitária no campo da assistência social.

Ao comparar funcionalidade (GMFCS) e fatores pessoais $($ FP*), que corresponde a curiosidade das crianças, foram encontradas diferenças significantes entre estas variáveis. Pode-se observar que $50 \%$ das crianças com comprometimento grau $\mathrm{V}(n=7)$ pontuaram negativo, possivelmente devido ao comprometimento das funções intelectuais (B 117) e funções mentais da linguagem (B 167). Há extensa literatura que evidencia que os diferentes domínios do desenvolvimento (motor, cognitivo e de linguagem) são interdependentes ao longo do tempo, ressaltando sua interação e capacidade preditiva de identificar atraso ${ }^{25,26}$. 
Foram limitações deste estudo prontuários com avaliações não padronizadas e número amostral que não viabilizou generalização dos resultados. Como a CIF não atribui uma única categoria para a condição de saúde, é possível a associação entre códigos, o que aumenta a complexidade da sua aplicação, que se tornará mais adequada à medida que for utilizada por mais profissionais da área da saúde, a partir de diferentes contextos.

\section{CONCLUSÃO}

Neste estudo, ao comparar a classificação da funcionalidade pelo instrumento GMFCS e as categorias descritas pelo modelo da CIF, pôde-se observar que os códigos do core set da CIF-CJ resumido para crianças e jovens com PC estão associados aos níveis de funcionalidade apresentados pelo GMFCS.

$\mathrm{Na}$ comparação entre funcionalidade (GMFCS) e funções do corpo (B), foram encontradas diferenças entre as cinco classificações funcionais e as funções intelectuais (B117), funções mentais da linguagem (B167), funções da mobilidade das articulações (B710), funções do tônus muscular (B735) e funções relacionadas ao controle dos movimentos voluntários (B760), onde quanto maior o comprometimento funcional identificado pela classificação GMFCS, maior o grau de comprometimento da deficiência dessas crianças. 
$\mathrm{Na}$ comparação entre funcionalidade (GMFCS) e atividades e participação (D), foram encontradas diferenças entre as cinco classificações funcionais e a manutenção da posição do corpo (D415), uso fino da mão (D440), andar (D450), deslocar-se por diferentes locais (D460), comer (D550), interações interpessoais básicas (D710) e relações familiares (D760), onde quanto maior o comprometimento funcional (maior grau na GMFCS), maiores eram as dificuldades apresentadas (ou relatadas) pelas crianças desta amostra nessas atividades e participações.

Na comparação entre funcionalidade (GMFCS) e fatores ambientais (E), foram encontradas diferenças entre as cinco classificações funcionais e produtos e tecnologias para uso pessoal na vida diária (E115), produtos e tecnologias para mobilidade e transporte pessoal em ambientes internos e externos (E120), e produtos e tecnologias usados em projetos, arquitetura e construção de edifícios para uso público (E150), onde quanto maior o comprometimento funcional, mais completos e graves eram os facilitadores.

Estes resultados evidenciam que quanto maior 0 comprometimento funcional identificado pala classificação GMFCS, maior o grau de comprometimento da deficiência dessas crianças nas categorias da CIF-CJ função do corpo, atividades e participação e fatores ambientais.

Os dois instrumentos são complementares na avaliação da criança e do adolescente com PC e podem respaldar o raciocínio clínico para tomada de decisões dos profissionais 
que os assistem, bem como nortear políticas públicas para a promoção da qualidade de vida dessa população.

\section{AGRADECIMENTOS}

Agradecimento à Associação Canoense de Deficientes Físicos (ACADEF) e aos fisioterapeutas Jivago Di Napoli e Karine Nitzel pela anuência para a realização desta pesquisa.

\section{REFERÊNCIAS}

1.Polita NB, Tacla MTGM. Rede e apoio social às famílias de crianças com paralisia cerebral. Esc Anna Nery Revista de Enfermagem 2014; 18:75-81. http://dx.doi.org/10.5935/1414-8145.20140011

2. Reitz GS, Chirolli MJ, Gantzel LC, Lunardelli BS, Pereira SM, Roesler $\mathrm{H}$. Efeitos da prática do suporte de peso corporal em crianças com paralisia cerebral: uma série de casos. Rev Pesq Fisioterap 2018;8:397-403. http://dx.doi.org/10.17267/2238-

2704rpf.v8i3.1982

3.Ferreira MC, Di Naccio BL, Otsuka MYC, Barbosa AM, Corrêa PFL, Gardenghi G. Avaliação do índice de sobrecarga de cuidadores primários de crianças com paralisia cerebral e sua relação com a qualidade de vida e aspectos socioeconômico. Rev Acta Fisiatr 2015;22:9-13. https://doi.org/10.5935/0104-7795.20150003

4.Oliveira AIA, Assis GJA, Garotti MF. Tecnologias no ensino de crianças com paralisia cerebral. Rev Bras Edu Esp 2014;20:85-102. https://doi.org/10.1590/S1413-65382014000100007

5.Graham H, Rosenbaum P, Paneth N, Dan B, Lin JP, Damiano DL, et al. Cerebral palsy. Nat Rev Dis Primers 2016;2:15082. https://doi.org/10.1038/nrdp.2015.82

6. Labaf S, Shamsoddini A, Hollisaz MT, Sobhani V, Shakibaee A. Effects of Neurodevelopmental Therapy on Gross Motor Function in Children with Cerebral Palsy. Iran J Child Neurol 2015;9:36-41. http://journals.sbmu.ac.ir/ijen/article/view/6165/7031

7. Herskind A, Greisen G, Nielsen JB. Early identification and intervention in cerebral palsy. Rev Dev Med Child Neurol 2014;57:2936. https://doi.org/10.1111/dmcn.12531

8.Silva DBR, Dias LB, Pfeifer LI. Confiabilidade do Sistema de Classificação da Função Motora Grossa Ampliado e Revisto (GMFCS E $\& R$ ) entre estudantes e profissionais de saúde no Brasil. Fisioter Pesq 2016;23:142-7. 
https://doi.org/10.1590/1809-2950/14396823022016

9.Santos PD, Silva FC, Ferreira EG, Iop RR, Bento GG, Silva R. Instrumentos que avaliam a independência funcional em crianças com paralisia cerebral: uma revisão sistemática de estudos observacionais. Fisioter Pesq 2016;23:318-28. https://doi.org/10.1590/18092950/15260723032016

10.Palisano R, Rosenbaum P, Walter S, Russel D, Wood E, Galuppi B. GMFCS - E \& R Sistema de Classificação da Função Motora Grossa Ampliado e Revisto. Hamilton: CanChild Centre for Childhood Disability Research, McMaster University. 2007. https://canchild.ca/system/tenon/assets/attachments/000/000/075/0 riginal/GMFCS-ER Translation-Portuquese2.pdf

11.Organização Mundial de Saúde. CIF: classificação internacional de funcionalidade, incapacidade e saúde. São Paulo: Editora USP, 2003.

12. Mukhtiar K, Ibrahim S, Ahmed M. Application of CF-CY Core Sets in Children with Spastic Cerebral Palsy. J Ped Neurol 2020;18:17-21. https://doi.org/10.1055/s-0039-1685157

13.Schiariti V, Selb M, Cieza A, O'Donnell M. International Classification of Functioning, Disability and Health Core Sets for children and youth with cerebral palsy: a consensus meeting. Dev Med Child Neurol 2015;57:149-58. https://doi.org/10.1111/dmcn.12551

14. Oliveira LS, Golin MO. Técnica para redução do tônus e alongamento muscular passivo: efeitos na amplitude de movimento de crianças com paralisia cerebral espástica. ABCS Health Sci 2017;42:27-33. https://doi.org/10.7322/abcshs.v42i1.946

15.Santos RA, Silva VR, Santos JP, Siqueira NA. Perfil epidemiológico e assistência à saúde de crianças e adolescentes com paralisia cerebral em um município do ES. Rev Resid Ped 2019;9:252-60. https://doi.org/10.25060/residpediatr-2019.v9n3-10

16.Guimarães CL, Pizzolatto TCO, Coelho ACS, Freitas STT. Aspectos clínicos epidemiológicos de crianças com paralisia cerebral assistidas pela clínica escola de Fisioterapia UNIP-São José dos Campos. J Health Sci Inst 2014;32:281-5.

https://www.unip.br/presencial/comunicacao/publicacoes/ics/edicoes /2014/03 jul-set/V32 n3 2014 p281a285.pdf

17. Ministério da Saúde. Secretaria de Atenção à Saúde. Departamento de Ações Programáticas Estratégicas. Diretrizes de atenção à pessoa com paralisia cerebral. Brasília: Ministério da Saúde, 2014. https://bvsms.saude.gov.br/bvs/publicacoes/diretrizes atencao paral isia cerebral.pdf

18.Gómez MES, Quintero LCB, Poveda IGG, Ortiz PVR, Jaramillo LFG, Gómez $M A B$, et al. Rasgos característicos del comportamiento motor del niño con prematurez durante los primeros meses de vida posnatal: Una revisión de la literatura. Rehabilitación 2020;54:31-40. https://doi.org/10.1016/j.rh.2019.09.004

19. Heathcock JC, Baranet K, Ferrante R, Hendershot S. Daily Intervention for Young Children With Cerebral Palsy in GMFCS Level V: A Case Series. Pediatr Phys Ther 2015;27:285-92. https://doi.org/10.1097/PEP.0000000000000149 
20.Garfinkle J, Wintermark P, Shevell MI, Oskou M. Cerebral palsy after neonatal encephalopathy: do neonates with suspected asphyxia have worse outcomes? Dev Med Child Neurol 2015;58:189-94. https://doi.org/10.1111/dmcn.12953

21.Xavier CL, Brito JNPO, Moura MEB, Abreu BAL, Brito ES. Condições de saúde da criança acometida por paralisia cerebral na Estratégia Saúde da Família. Rev Pesq Cuidado Fund 2014;6:22-33. https://www.redalyc.org/pdf/5057/505750772003.pdf

22.Verdiani MB, Gomes JL, Nishida MH, Marinho MP, Braga DM. Aplicabilidade da CIF baseada nos objetivos funcionais na Paralisia Cerebral. Rev Cien CIF Brasil 2016;5:2-14. http://www.revistacifbrasil.com.br/ojs/index.php/CIFBrasil/article/vie $\mathrm{w} / 30$

23.Ferreira HNC, Cirne GNM, Pereira AS, Lima NMFV, Cacho RO, Cacho EWA. Upper extremity motor quality evaluation in children with Cerebral Palsy. Fisioter Mov 2017;30:277-84. https://doi.org/10.1590/19805918.030.s01.a027

24.Souza NP, Alpino MAS. Avaliação de Crianças com Diparesia Espástica Segundo a Classificação Internacional de Funcionalidade, Incapacidade e Saúde (CIF). Rev Bras Edu Esp 2015;21:199212. https://doi.org/10.1590/S1413-65382115000200003

25.Borba LS, Pereira KRG, Valentini NC. Motor and cognitive development predictors of infants of adolescents and adults mothers. J Phys Edu 2017;28:1-16. https://doi.org/10.5007/1980$0037.2015 v 17 n 4 p 438$

26.Maia TSA, Silva Júnior RA, Silva ER, Medeiros C, Wanderley Filho HM. Avaliação da função motora grossa em pacientes com encefalopatia crônica não progressiva da infância com o uso da suit terapia. Fisiot Brasil 2018;19:33-42. 